\title{
Increased p53 Protein Expression in Malignant Mammary Phyllodes Tumors
}

\author{
Gary M.K. Tse, FRCPC, Thomas C. Putti, Dip. Am. Bd. (Path.), Fred Y.L. Kung, B.Sc., \\ Richard A. Scolyer, FRCPA, Bonita K.B. Law, FRCS, Tai-shing Lau, Ph.D., C. Soon Lee, FRCPA \\ Departments of Anatomical and Cellular Pathology (GMKT, FYLK), Surgery (BKBL), Prince of Wales \\ Hospital, and Statistics (T-SL), Chinese University of Hong Kong, Hong Kong; Department of Pathology, \\ National University Hospital (TCP), Singapore; and Department of Pathology, University of Sydney, and \\ Department of Anatomical Pathology, Royal Prince Alfred Hospital (RAS, CSL), Sydney, Australia
}

The authors reviewed 143 cases ( 87 benign, 37 borderline, and 19 malignant) of mammary phyllodes tumors (PTs) and used immunohistochemistry to detect p53 protein product semi-quantitatively as negative, weak, moderate and strong (scored 0 to 3 ). For all PTs, an increasing trend of tumor size and malignancy was detected with increasing age. For p53 staining, 60 cases $(42 \%)$ were negative, 55 (38\%) stained weakly, 28 (13\%) stained moderately, and $10(7 \%)$ stained strongly. Of the 87 benign PTs, 41 (47\%) were negative, 37 (43\%) stained weakly, and 9 (10\%) stained moderately. For the 37 borderline PTs, $16(43 \%)$ were negative, $14(38 \%)$ stained weakly, 6 (16\%) stained moderately, and $1(3 \%)$ stained strongly. Of the 19 malignant PTs, $3(16 \%)$ were negative, $4(21 \%)$ stained weakly, $3(16 \%)$ stained moderately, and 9 (47\%) stained strongly. The mean intensity score for p53 staining increased progressively from benign to borderline to malignant PT, with established statistical significance ( $P$ $<.0001$ ). This is significantly correlated with mitotic count but not stromal cellularity, pleomorphism, margin, and stromal overgrowth. When considering strong staining alone (score, 3), $47 \%$ of malignant, $3 \%$ of borderline, and none of the benign PTs were positive. The use of strong positive staining for diagnosing malignant PT gave positive and negative predictive values, specificity, and sensitivity of $90 \%$, $92.5 \%, 99 \%$, and $47 \%$, respectively. Thus diffuse strong p53 protein staining can be used as a soft sign in assisting the diagnosis of malignant PT. Con-

Copyright (C) 2002 by The United States and Canadian Academy of Pathology, Inc.

VOL. 15, NO. 7, P. 734, 2002 Printed in the U.S.A.

Date of acceptance: April 3, 2002.

Address reprint requests to: Gary M.K. Tse, Senior Medical Officer, Department of Anatomical and Cellular Pathology, Prince of Wales Hospital, Ngan Shing Street, Shatin, NT, HKSAR, China; e-mail: garytse@cuhk.edu.hk; fax: 852-26374858.

DOI: 10.1097/01.MP.0000018978.75312.5C versely, negative or weak staining of $\mathrm{p53}$ protein in PT is of little discriminatory value. The role of p53 gene mutation in the malignant transformation of PT is unclear; but this may not be the sole mechanism as many malignant $\mathrm{PT}$ were $\mathrm{p53}$ protein negative.

KEY WORDS: Breast, Immunohistochemistry, Malignant, p53, Pathology, Phyllodes tumor. Mod Pathol 2002;15(7):734-740

Phyllodes tumor (PT) is an uncommon stromalepithelial neoplasm of the breast. The reported incidence is 0.3 to $0.5 \%$ of female breast tumors (1-5). The median and mean age of patients is $45 \mathrm{y}$, and the average size is $4-5 \mathrm{~cm}$. Rarely, these lesions can occur in younger and older women and in men. Clinically, PT is difficult to distinguish from fibroadenoma. The histological grading of PT is based on a combination of histological features, including mitotic count, cellularity, and pleomorphism of the stromal cells, stromal overgrowth, and whether the border is infiltrative or not $(2,6)$. By using these parameters, PT is divided into benign, borderline, and malignant. Although both borderline and malignant PT can metastasize, all PTs can recur locally. This propensity to recur makes proper and adequate treatment imperative, even in benign cases.

Mutations of the p53 tumor suppressor gene are among the commonest detected in human malignancies (7). Accumulation of the protein product, as detected by immunohistochemistry, has been described in many tumor types as a marker of neoplastic progression and of aggressiveness $(8,9)$. In the literature, evaluation of p53 protein expression in PT has been reported in several studies (10-14). In the studies that correlated the grade of PT to p53 protein expression, it was suggested that malignant PT have diffuse strong staining that allowed distinction of malignant from benign and borderline PT 
(10-12). Other studies suggested that p53 protein expression did not predict outcome $(11,13)$. In the current study, we further evaluated the relationship of p53 protein expression with histologic parameters and the role of p53 protein detection in the diagnosis of malignancy in PT.

\section{MATERIALS AND METHODS}

The histopathology files from the three participating departments were searched for PT over the past 14 years, yielding a total of 143 cases. The paraffin blocks were retrieved and $4-\mu \mathrm{m}$ slides prepared routinely, stained with hematoxylin and eosin. All the slides were reviewed for the following histologic parameters: (1) stromal cellularity; (2) nuclear pleomorphism; (3) stromal overgrowth; (4) mitotic count; and (5) margin of the tumor, whether infiltrative or rounded. The stromal cellularity and nuclear pleomorphism were graded as low/mild, moderate, or severe; stromal overgrowth was graded as mild, moderate (scanty epithelial element within a low-power field), or severe (absence of epithelial element within a low-power field [40X]; Nikon Labophot; field area, $1.9 \mathrm{~mm}^{2}$ ); and the mitotic count was given as the number of mitotic figures per 10 high-power fields $(400 \times$; Nikon Labophot; field area, $0.19 \mathrm{~mm}^{2}$ ). A diagnosis of benign PT was made when there was low cellularity, no stromal overgrowth, mild pleomorphism, a rounded margin, and a mitotic count of two orless per 10 high-power fields. Malignant PT was diagnosed when the mitotic count was five or more per 10 high-power fields, together with stromal overgrowth and an infiltrative margin. Borderline PT was diagnosed when the criteria for malignancy were not totally fulfilled.

For p53 staining, a most-representative slide was taken from each case and stained for p53 (DO-7 monoclonal, Novocastra, UK) using standard avidin-biotin method with microwave antigen retrieval. The staining of cells was assessed according to both the intensity and proportion of positive cells. The staining pattern was graded from 0 to 3 , with 0 being no staining; 1 when $<33 \%$ of the stromal cell nuclei stained weakly; 2 when $34-67 \%$ of cell nuclei stained with weak to moderate staining intensity; and 3 when $>67 \%$ cells displayed moderate to strong nuclear staining.

For statistical analysis, PROC LOGISTIC in SAS was used to study the association between p53 and diagnosis with the presence of the five histologic factors and recurrence. Student $t$ test and ANOVA was used to compare the tumor size and patient age with diagnosis of PT and also between the recurrent and nonrecurrent tumors. Statistical significance is established at $P<.05$.

\section{RESULTS}

One hundred and thirty-seven patients were included in this study, including four patients with both the initial and first recurrent PT, one patient with the initial and two additional recurrences, six patients with only the first recurrence, one patient with only the second recurrence, and the initial PT of two patients with one recurrence, yielding a total of 143 PTs. The patient's age ranged from 15 to 77 years (mean, $43 \mathrm{y}$ ), and the tumor size ranged from 1 to $22 \mathrm{~cm}$ (mean, $5 \mathrm{~cm}$ ). Sixty-two percent of the patients were Chinese, $21 \%$ were Caucasian, and the remainder were Indo-Chinese or Indian. The tumors occurred on the left side in $51 \%$ of cases, and $48 \%$ occurred on the right; in two cases, the side was not known. Of the total cases, there were $87(61 \%)$ benign, 37 (26\%) borderline, and $19(13 \%)$ malignant PT.

For the 87 benign PT, the patient age range was 17 to 62 years (mean, $40.8 \mathrm{y}$ ), and the tumor size range was 1 to $22 \mathrm{~cm}$ (mean, $4.3 \mathrm{~cm}$ ). For the 37 borderline PT, the patient age range was 15 to 77 years (mean, $44.6 \mathrm{y}$ ), and the tumor size range was 1 to $20 \mathrm{~cm}$ (mean, $5.8 \mathrm{~cm}$ ). For the 19 malignant PT, the patient age range was 30 to 76 years (mean, $49.8 \mathrm{y})$, and the tumor size range was 2 to $22 \mathrm{~cm}$ (mean, $6.8 \mathrm{~cm}$ ). The mean age of patients increased with the degree of malignancy of PT. The difference between age of patients of the benign and malignant groups was significant $(P=.0008)$, but no statistical significance was demonstrated between the benign and borderline groups and between the borderline and malignant groups. The sizes of the PTs also increased with increasing degree of malignancy, but the differences were not statistically significant.

For the p53 protein staining of all cases, 60 cases (42\%) scored 0; 55 cases (38\%) scored 1; 18 cases (13\%) scored 2 (Figs. 1 and 2); and 10 cases (7\%) scored 3 (Fig. 3 and 4). In the 60 cases with score 0 , $41(68 \%)$ were benign, $16(27 \%)$ were borderline, and $3(5 \%)$ were malignant. In the 55 cases with score 1, $37(67 \%)$ were benign, $14(25 \%)$ were borderline, and $4(8 \%)$ were malignant. For the 18 cases with score 2, $9(50 \%)$ were benign, $6(33 \%)$ were borderline, and $3(17 \%)$ were malignant. For the 10 cases with score 3,1 (10\%) was borderline and 9 (90\%) were malignant.

For the 87 benign PTs, 41 cases (47\%) scored 0, 37 cases $(43 \%)$ scored 1 , and 9 cases $(10 \%)$ scored 2. The mean score for benign PTs was 0.63 . For the 37 borderline PTs, 16 cases $(43 \%)$ scored 0,14 cases (38\%) scored 1, 6 cases (16\%) scored 2, and 1 case 


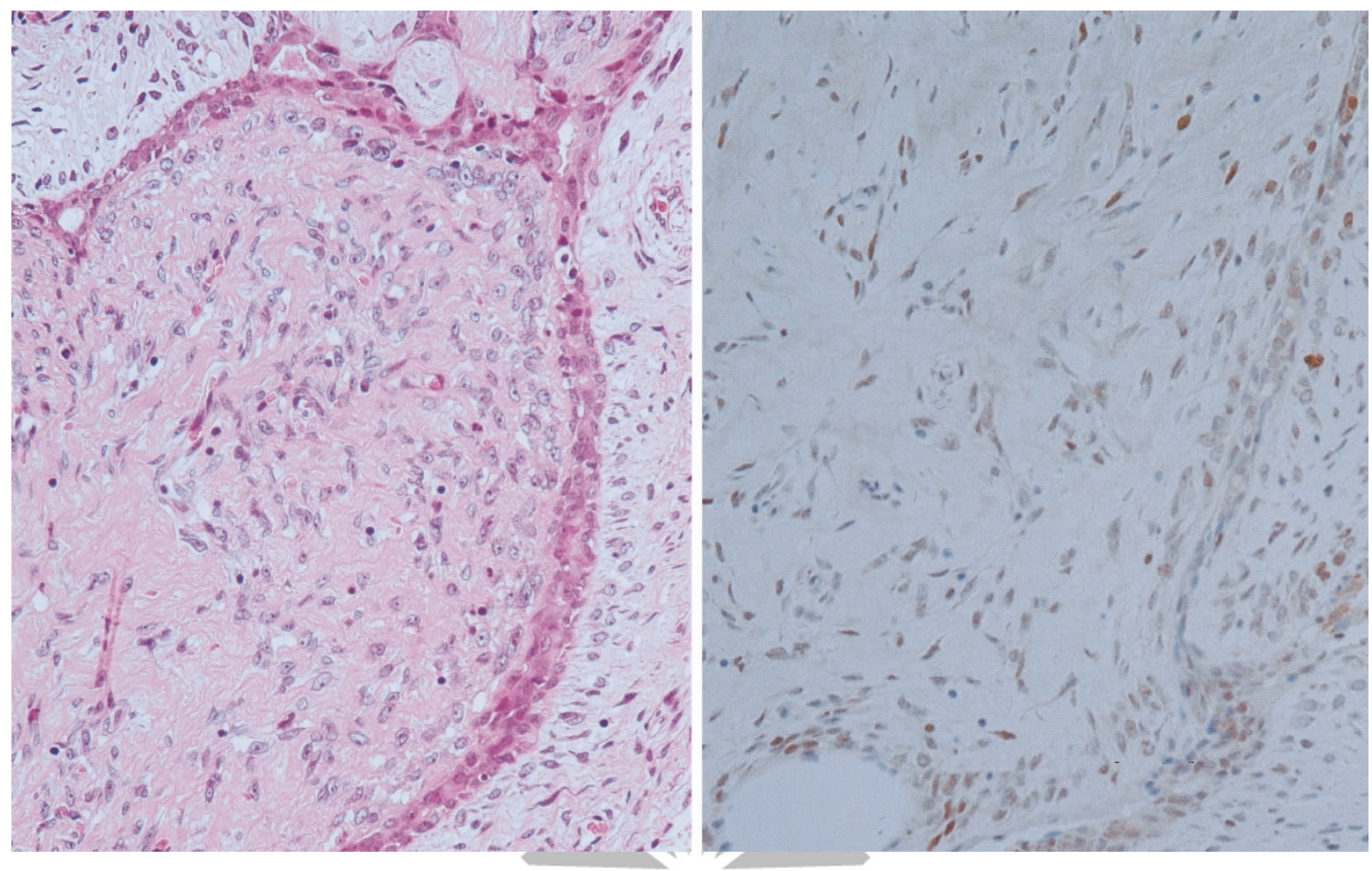

FIGURE 1. Photomicrograph showing a borderline phyllodes tumor (hematoxylin and eosin, $200 \times$, left panel) with moderate (34-67\%) p53 staining of stromal cell nuclei $(200 \times$, right panel $)$.
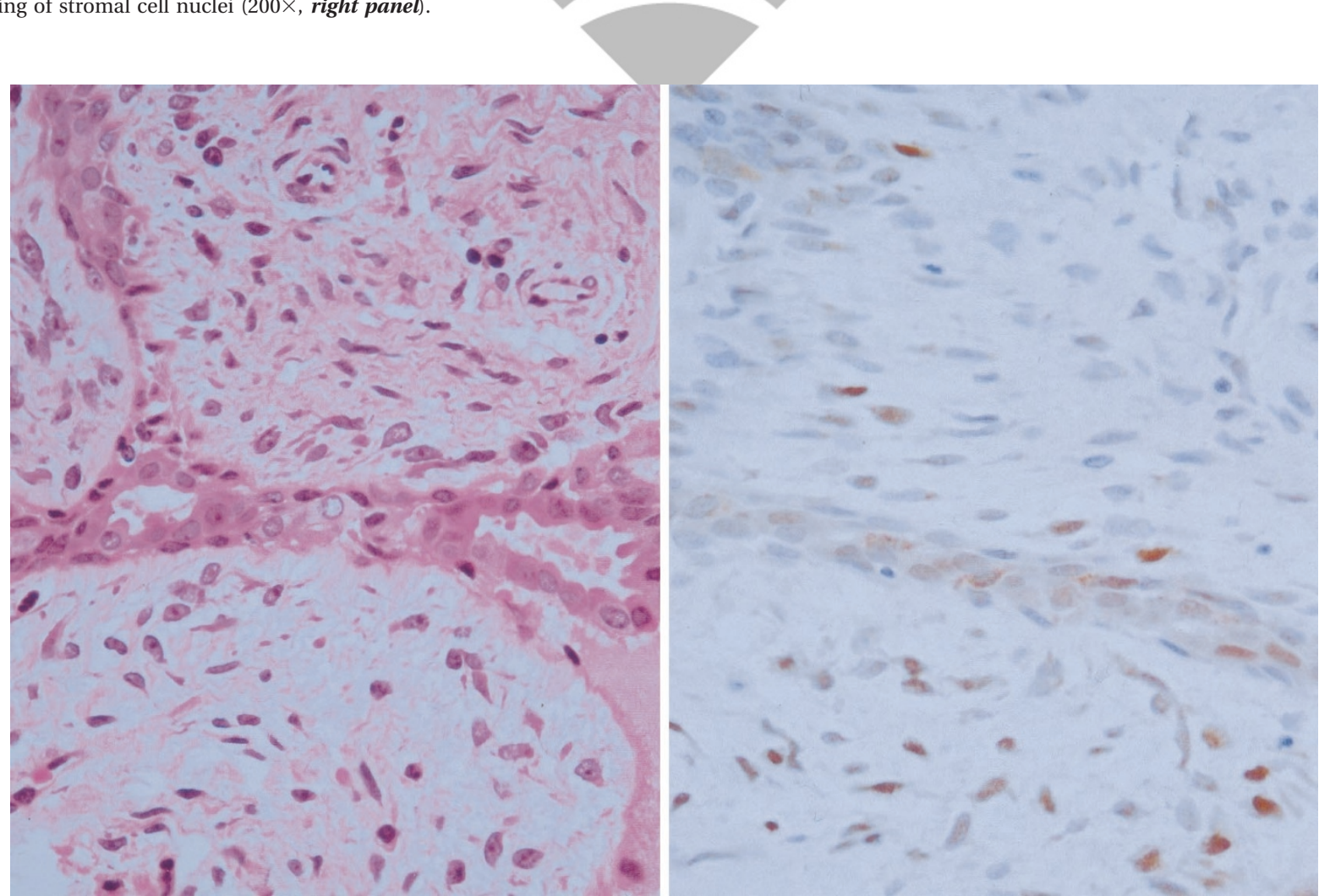

FIGURE 2. Photomicrograph showing a borderline phyllodes tumor (hematoxylin and eosin, $400 \times$, left panel) with moderate (34-67\%) p53 staining of stromal cell nuclei $(400 \times$, right panel).

(3\%) scored 3. The mean score for borderline PTs was 0.78 . For the 19 malignant PTs, 3 cases $(16 \%)$ scored 0,4 cases $(21 \%)$ scored 1,3 cases $(16 \%)$ scored 2, and 9 cases (47\%) scored 3 . The mean score for malignant PTs was 1.95. Using ANOVA, the scores were significantly different among the three groups $(P<.0001)$ when considering the diagnosis with p53 score. 


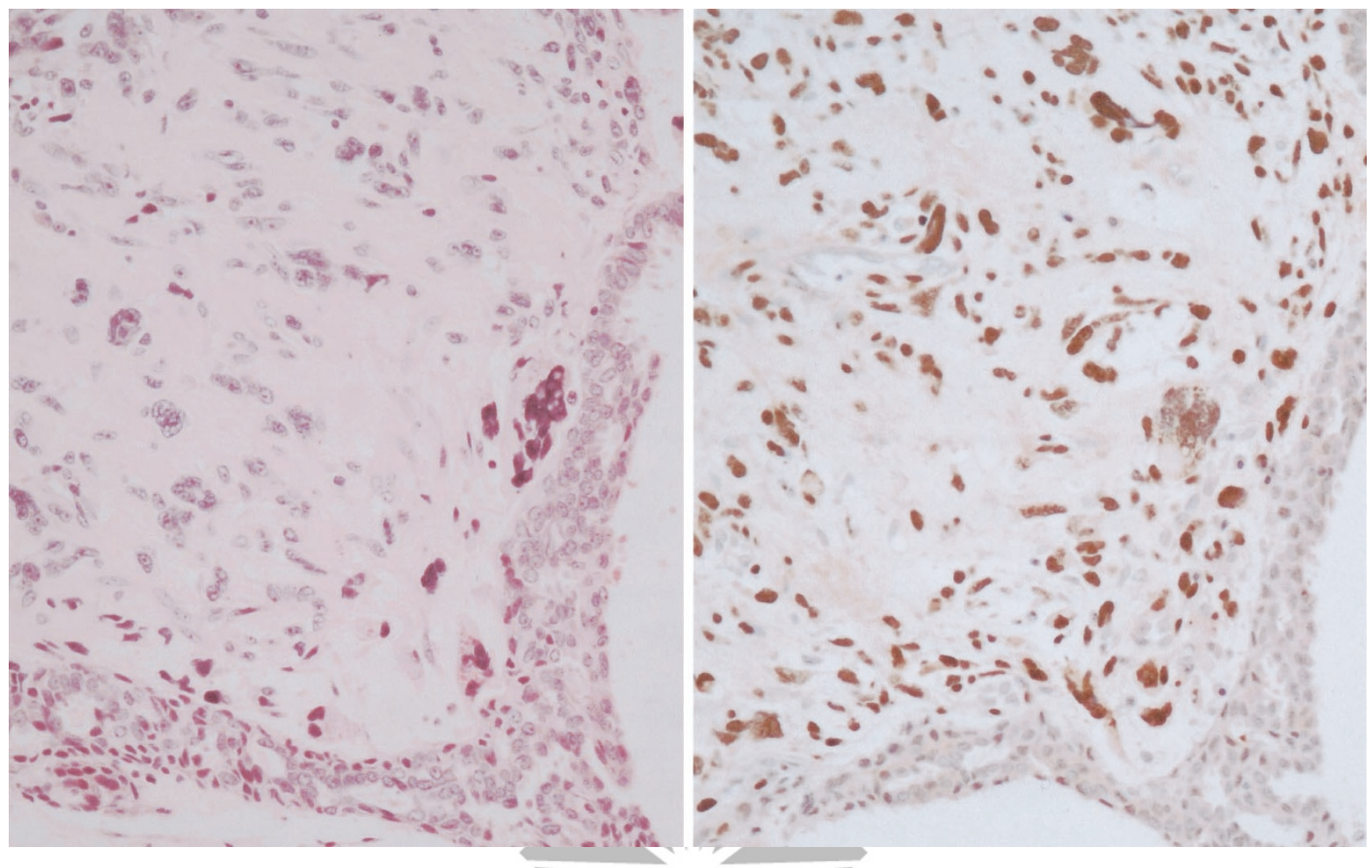

FIGURE 3. Photomicrograph showing a malignant phyllodes tumor (hematoxylin and eosin, $200 \times$, left panel) with diffuse strong ( $>67 \%$ ) p53 staining of the stromal cell nuclei $(200 \times$, right panel $)$.

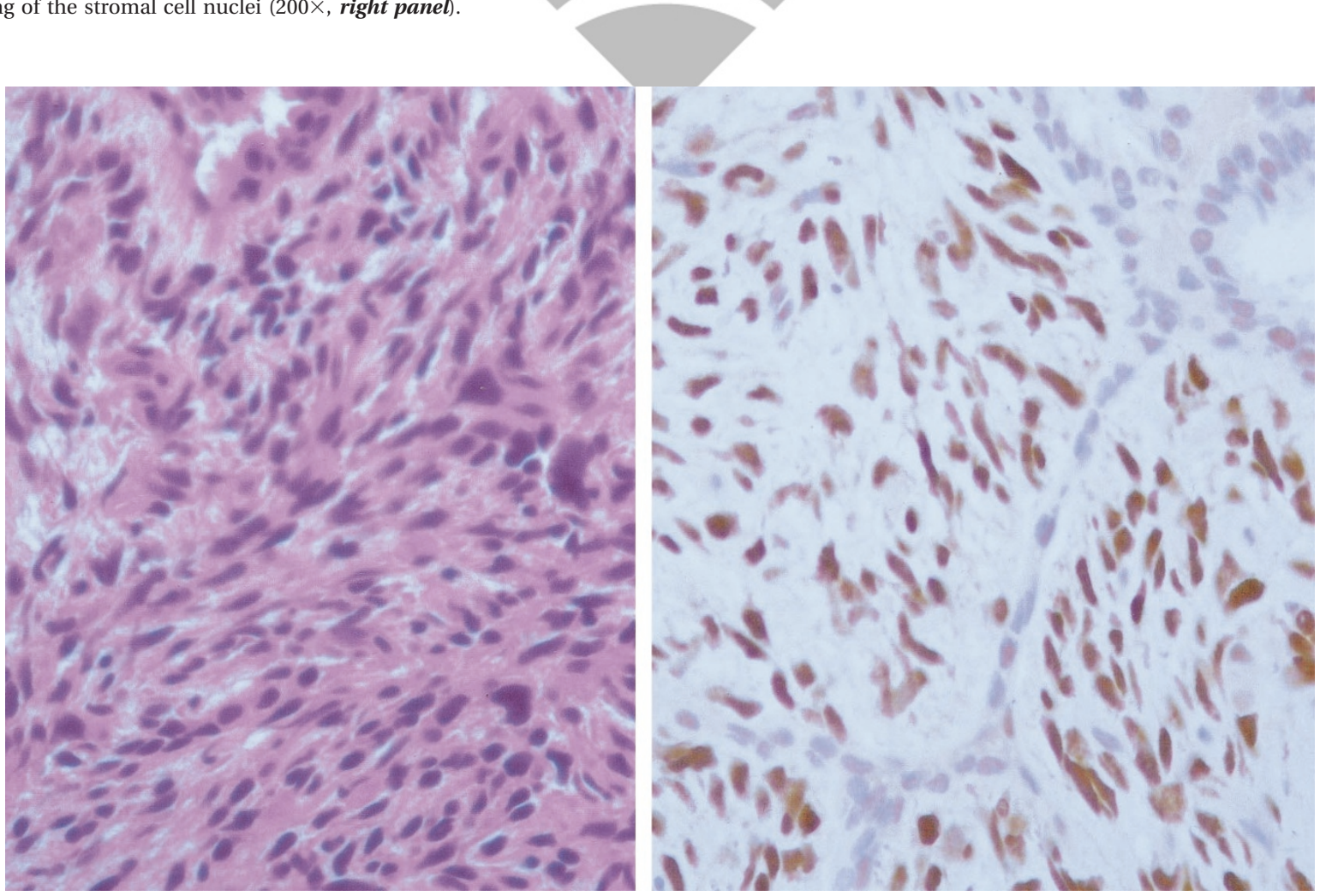

FIGURE 4. Photomicrograph showing a malignant phyllodes tumor (hematoxylin and eosin, $400 \times$, left panel) with diffuse strong ( $>67 \%$ ) p53 staining of the stromal cell nuclei $(400 \times$, right panel).

We further analyzed whether the p53 score was related to any of the histologic parameters or whether the p53 score was independent of these parameters. A logistic regression model was used, taking into consideration the presence of the five histologic variables that were used for establishment of diagnosis. The correlation of p53 score with diagnosis now became statistically not significant 
$(P=.187)$. Of the five histologic parameters analyzed, four of them (cellularity, nuclear pleomorphism, stromal overgrowth, and margin pattern) were not associated with p53 score; only mitotic count showed a relationship with p53 score $(P=$ .0309). In fact, if we remove the mitotic count in the first logistic regression model, the association between p53 and diagnosis became significant $(P=$ .0055), indicating that the information given by p53 and mitotic count were overlapping.

Of the 137 patients, 17 were lost to follow-up. For the remaining 120 patients, the follow-up period starting from the occurrence of the initial PT ranged from 2 to 300 months, with a mean of 68 months. One hundred patients were well and did not have any recurrence, 18 had recurrences, and 2 had distant metastases. For the 18 patients with recurrences, 14 had one recurrence, and 4 had two recurrences. If only the first recurrences were considered, the recurrence interval after the initial tumor was 6 to 204 months, with a mean of 36 months. One case had a recurrence interval of 204 months, and for all others, the recurrence occurred within 72 months. For the second recurrence, the range was 24 to 240 months (mean, $91.8 \mathrm{mo}$ ). All the second recurrences occurred within 12 to 75 months after the first recurrence. For the disease outcome, one of the two patients with distant metastases died 2 years after diagnosis. For these two patients, one did not have histologic assessment as the metastases were diagnosed radiologically. For the other case, the metastasis was composed of epithelioid malignant stromal cells with brisk mitoses. All other patients were alive at the end of the follow-up period.

Among the recurrences, six recurrent tumors (five first recurrences and one second recurrence from five patients) together with the initial PT were available for review. The initial tumor was benign in one, borderline in two, and malignant in two cases. The benign PTs recurred as benign, but the recurrences from the borderline and malignant groups recurred as all categories. No definite relationship could be established between the grade of the initial and the recurrent tumors. Analysis of the histologic variables and p53 scores using logistic regression showed that most of these are not predictive of the occurrence of recurrence. Only the mitotic count was related to recurrence $(P=.0062)$.

When we compared the histologic parameters, tumor sizes, and p53 protein expressions of the recurrences and the nonrecurrent PTs using the $t$ test, the recurrences had higher degree of nuclear pleomorphism, cellularity, stromal overgrowth and mitoses, but these were not statistically significant $(P=.1076-.2305)$.

\section{DISCUSSION}

The division of PTs into benign, borderline, and malignant is essentially arbitrary because these lie along a histologic continuum rather than discreet histologic categories. Classification into benign and malignant PTs uses a combination of histologic criteria, and for cases that fulfill some but not all malignant criteria, they are labeled as of borderline malignancy. This continuum is well illustrated by the fact that in different large series, the proportion of borderline PTs differed significantly, ranging from 11 to $42 \%$, with the malignant PTs ranging from 5 to $45 \%$ (13-18). The findings in the current series fell within these ranges. This division is nevertheless important because malignant PT has higher potential to metastasize. Additional factors that have been studied to assist in the differentiation of different categories of PT included p53, CD34, bcl-2, Ki-67, endothelin 1, Factor XIIIa, and microvessel density (19-23).

Studies evaluating p53 protein expression in PT are few in the literature. Most of the series included limited number of cases $(<20)$ of PT $(10,12,14)$, whereas two studies were more comprehensive and included 57 (11) and 118 (13) cases, respectively. Some of the smaller series also included only benign and malignant but not borderline PT in the analysis. The results of p53 protein expression from these studies were variable. In some studies, p53 protein expression was exclusively present in malignant PT but not in benign PT or fibroadenomas $(10,12)$, and some authors further distinguished the immunostaining patterns of weak and diffuse strong positivity (12). In other series with more cases and including borderline PT, the findings were less clear-cut, showing p53 protein expression in malignant, borderline (11), and even benign (13, 14) PT. In all these studies, the percentage of PTs with p53 protein expression differed between different grades of malignancy, ranging from 14 to $86 \%$ in malignant PTs, to 18 to $25 \%$ in borderline PTs, and to 0 to $10 \%$ in benign PTs $(11,14)$. In all individual studies, the percentage of p53 protein expression increased with ascending degree of malignancy.

Our results demonstrated several findings. First, we showed that with increasing degree of malignancy, a concurrent increase in p53 protein expression was detected, both in terms of the intensity and the percentage of cells. This observation is in agreement with the findings in other series (10-14).

We also demonstrated that p53 protein expression correlated strongly with the mitotic count but not with the diagnosis in the presence of mitotic count. This indicated that p53 expression overlapped with mitoses and was not an independent factor in predicting malignancy in PTs. This is ex- 
pected as the mechanism of p53 overexpression is related to controlled apoptosis in response to DNA damage. As was previously demonstrated in a smaller series (11), our results showed that p53 protein expression was statistically significantly related to the mitotic activity of the phyllodes tumor but did not predict outcome in terms of recurrence or mortality. Although other authors have reported an association of p53 expression with most histologic features (stromal overgrowth, nuclear pleomorphism, infiltrative margin), this was not observed in our study.

Immunostaining for p53 protein expression can be of utility in the diagnosis of the malignant cases. If only diffuse strong positivity for p53 protein expression is considered, almost half of the malignant PTs exhibited this high level of expression, whereas only $3 \%$ of the borderline and none of the benign PTs showed such strong expression. This finding echoes those of other authors (10-14), who demonstrated strong positive staining for malignant PTs. For the larger series that included borderline cases $(11,13,14)$, the percentage of positivity of borderline tumors ranged from 0 to $25 \%$, compared with a single case of a total of 37 cases (3\%) in the current series. The specificity of diffuse strong p53 protein staining in malignant PTs in our series is 99\%; sensitivity, $47 \%$; positive predictive value, $90 \%$; and negative predictive value, $92.5 \%$. This indicates that strong diffuse p53 staining is useful and specific in diagnosing malignant PTs with very high positive and negative predictive values.

In predicting recurrences, we showed that most histologic criteria and p53 protein expression were not useful, with only mitotic count correlating with recurrences. In comparing the recurrences and the nonrecurrent tumors, the recurrent tumors showed higher degree of stromal cellularity, pleomorphism, overgrowth, and mitoses, but these were not statistically significant. We believe that if more cases of recurrent tumors were available, statistical significance may be established for these parameters, which are at best weak predictors for recurrence. p53 protein expression is not useful in predicting outcome.

The underlying molecular mechanism of causation and progression of PTs remains poorly understood. In the literature, a few cytogenetic studies by comparative genomic hybridization (CGH) showed $3 \mathrm{p}$ loss and $1 \mathrm{q}$ gain to be the more common abnormalities in PTs $(24,25)$; however, these changes were not consistent. The role of p53 mutation in the malignant transformation of PTs has yet to be elucidated, although point mutation has been found to be the responsible mechanism in a case of a benign PT that transformed into a malignant PT (26). It would appear that with the strong and diffuse staining for p53 protein expression present in mostly malignant but not benign or borderline PTs, such mutations occur as late events in tumor progression. Furthermore, the presence of p53 protein expression in only some of the malignant PTs in all series suggests that changes in other tumor suppressor genes may also play a significant role in the malignant transformation. This may also partly explain the lack of power of p53 protein expression in predicting recurrences and outcome. Although the use of diffuse strong staining for p53 protein can be used as a "soft sign" in the diagnosis of malignant PT, further molecular studies are warranted to elucidate the mechanism, including that of p53 mutations, involved in the pathogenesis and progression of PT.

\section{REFERENCES}

1. Cole-Beuglet C, Soriano R, Kurtz AB, Meyer JE, Kopans DB, Goldberg BB. Ultrasound, X-ray mammography, and histopathology of cystosarcoma phyllodes. Radiology 1983;146: 481-6.

2. Azzopardi JG. Sarcoma of the breast. In: Bennington J, editor. Problems in breast pathology. Vol II. Major problems in Pathology. Philadelphia: Saunders; 1979. p. 355-9.

3. Palmer ML, De Risi DC, Pelikan A, Patel J, Nemoto T, Rosner $\mathrm{D}$, et al. Treatment options and recurrence potential for cystosarcoma phyllodes. Surg Gynecol Obstet 1990;170:193-6.

4. Kario K, Maeda S, Mizuno Y, Makino Y, Tankawa H, Kitazawa S. Phyllodes tumor of the breast: a clinicopathologic study of 34 cases. J Surg Oncol 1990;45:46-51.

5. Rowell MD, Perry RR, Hsiu JG, Barranco SC. Phyllodes tumors. Am J Surg 1993;165:376-9.

6. Rosen PP. Breast pathology. 2nd ed. Philadelphia: Lippincott-Raven; 2001. p. 176-97.

Lane DP. p53 and human cancers. Br Med Bull 1994;50:58299.

8. Carder P, Wyllie AH, Purdie CA, Morris RG, White S, Piris J, et al. Stabilised p53 facilitates aneuploid clonal divergence in colorectal cancer. Oncogene 1993;8:1397-401.

9. Neshat K, Sanchez CA, Galipeau PC, Blount PL, Levine DS, Joslyn G, et al. P53 mutations in Barrett's adenocarcinoma and high-grade dysplasia. Gastroenterology 1994;106:158995.

10. Millar EK, Beretov P, Marr P, Sarris M, Clarke RA, Kearsley $\mathrm{JH}$, et al. Malignant phyllodes tumours of the breast display increased stromal p53 protein expression. Histopathology 1999;34:491-6.

11. Feakins RM, Mulcahy HE, Nickols CD, Wells CA. p53 expression in phyllodes tumours is associated with histological features of malignancy but does not predict outcome. Histopathology 1999;35:162-9.

12. Kim CJ, Kim WH. Patterns of p53 expression in phyllodes tumors of the breast-an immunohistochemical study. J Korean Med Sci 1993;8:325-8.

13. Niezabitowski A, Lackowska B, Rys J, Kruczak A, Kowalska T, Mitus J, et al. Prognostic evaluation of proliferative activity and DNA content in the phyllodes tumor of the breast: immunohistochemical and flow cytometric study of 118 cases. Breast Cancer Res Treat 2001;65:77-85.

14. Kuenen-Boumeester V, Henzen-Logmans SC, Timmermans MM, van Staveren IL, Van Geel A, Peeterse HJ, et al. Altered expressiopn of p53 and its regulated proteins in phyllodes tumours of the breast. J Pathol 1999;189:169-75.

15. Shabalova IP, Chemeris GJ, Ermilova VD, Rodionova LM, Pavlikova NA, Syrjanen KJ. Phyllodes tumour: cytologic and 
histologic presentation of 22 cases, and immunohistochemical demonstration of p53. Cytopathology 1997;8:177-87.

16. Suo Z, Nesland JM. Phyllodes tumor of the breast: EGFR family expression and relation to clinicopathological features. Ultrastruct Pathol 2000;24:371-81.

17. Reinfuss M, Mitus J, Duda K, Stelmach A, Rys J, Smolak K. The treatment and prognosis of patients with phyllodes tumor of the breast: an analysis of 179 cases. Cancer 1996;77:910-6.

18. Cohn-Cedermark G, Rutqvist LE, Rosendahl I, Silfversward C. Prognostic factors in cystosarcoma phyllodes. A clinicopathologic study of 77 patients. Cancer 1991;68:2017-22.

19. Moore T, Lee AHS. Expression of CD34 and bcl-2 in phyllodes tumours, fibroadenomas and spindle cell lesions of the breast. Histopathology 2001;38:62-7.

20. Silverman JS, Tamsen A. Mammary fibroadenoma and some phyllodes tumour stroma are composed of CD34+ fibroblasts and factor XIIIa dendrophages. Histopathology 1996; 29:411-9.

21. Yamashita J, Ogawa M, Egami H, Matsuo S, Kiyohara H, Inada $\mathrm{K}$, et al. Abundant expression of immunoreactive endothelin 1 in mammary phyllodes tumor: possible paracrine role of endothelin 1 in the growth of stromal cells in phyllodes tumor. Cancer Res 1992;52:4046-9.

22. Gatalica Z, Lucio E, Finkelstein S, Palazzo J, Tawlik O. The role of p53 mutation and Ki-67 proliferation index in the diagnosis and progression of phyllodes tumor of the breast [abstract]. Lab Invest 1999;79:21A.

23. Tse GM, Ma TK, Chan KF, Law BK, Chen MH, Li KH, et al. Increased microvessel density in malignant and borderline mammary phyllodes tumours. Histopathology 2001;38:67-70.

24. Sawyer EJ, Hanby AM, Ellis P, Lakhani SR, Ellis IO, Boyle S, et al. Molecular analysis of phyllodes tumors reveals distinct changes in the epithelial and stromal components. Am J Pathol 2000;156:1093-8.

25. Woolley PV, Gollin SM, Riskalla W, Finkelstein S, Stefanik D, Riskalla L, et al. Cytogenetics, immunostaining for fibroblastic growth factors, p53 sequencing and clinical features of two cases of cystosarcoma phyllodes. Mol Diagn 2000;5:17990.

26. Gatalica Z, Finkelstein S, Lucio E, Tawfik O, Palazzo J, Hightower B, et al. p53 protein expression and gene mutation in phyllodes tumors of the breast. Pathol Res Pract 2001;197: 183-7.

\section{Book Review}

Gospodarowicz MK, Henson DE, Hutter RVP, $O$ 'Sullivan B, Sobin LH, Wittekind Ch, editors: Prognostic Factors in Cancer, 2nd ed, 832 pp, New York, Wiley-Liss, 2001 (\$99.95).

Sir William Osler, quoted in the preface of this book, wrote in 1904 that 'medicine is a science of uncertainty and an art of probability.'This book, sponsored by the International Union Against Cancer (UICC), shows how successful we have been in removing uncertainty from the science and making probability prediction more science than art.

The first 150 or so pages are devoted to theory of clinical prognostic factors. Nice, probably important and laudable, but unfortunately not my cup of tea. For most clinical oncologists, the remaining 600 pages will be of greater interest. Page by page, chapter by chapter (35 in total), the experts deal with the problems encountered on a daily basis in the practice of oncology. The fact that the roster of editors and contributors contains the names of several pathologists is in itself a sign that it should be of interest to pathologists as well. And indeed it is. Most chapters (except the first few) are peppered with pathology data. 'Our' contributions range from classical histopathology to molecular biology; from established, generally accepted facts to investigational results that still need to be validated on a broader base. Clinical-pathologic correlations are a prominent feature of every chapter and are generally well done.
The contributors hail from various parts of the world, reflecting the international nature of the IUCC. The editing of such contributions must be a gargantuan job, and although the overall result is enviably good, there are still significant disparities between the various chapters. Personally $\mathrm{I}$ would have appreciated a more structured and a 'predictably uniform' approach, which I feel should be mandated for all chapters in the future editions. Nevertheless, the positive aspects of the book definitely outweigh the few negative ones.

I have used the book and found it most useful while preparing for the interdisciplinary oncology conferences and meetings with clinicians. The reason-because it allowed me to review the relevant clinical prognostic data in the shortest possible time frame. I found it also authoritative and dependable. In some aspects it is not exactly as up to date as one would have wished it to be, but that is inevitable for a discipline that is advancing as fast as oncology. It is a work in progress, which I predict will improve with every new edition. The contributors and especially the editors deserve kudos for their concentrated effort to 'to provide the framework for the. . .application of prognostic factors in clinical decision making' in oncology.

\section{Ivan Damjanov \\ University of Kansas School of Medicine \\ Kansas City, Kansas}

